\title{
Pacientes internados em unidade de terapia intensiva que não adotam postura antigravitacional apresentam maiores chances de óbito
}

Patients admitted to an intensive care unit who do not adopt an antigravity posture have a higher odds of death

Pacientes hospitalizados en unidad de cuidados intensivos que no adoptan postura antigravitacional pueden tener mayores posibilidades de fallecimiento

Gilmara Oliveira Santos', Rodrigo Santos de Queiroz ${ }^{2}$, Cleber Souza de Jesus ${ }^{3}$, José Ailton Oliveira Carneiro ${ }^{4}$, Luciano Magno de Almeida Faria ${ }^{5}$, Marcos Henrique Fernandes ${ }^{6}$, Janilson Matos Teixeira Matos ${ }^{7}$

RESUMO | Ainda há poucos marcadores de desempenho funcional com capacidade de predizer óbito em unidades de terapia intensiva (UTI). O objetivo do presente estudo foi identificar a associação entre a não adoção de postura antigravitacional e óbito em pacientes internados em uma UTI adulto. Trata-se de um estudo retrospectivo e analítico, realizado através da análise de prontuários. A associação entre a não adoção de postura antigravitacional e óbito foi testada por regressão logística múltipla ajustada por sexo, idade, gravidade da doença (mensurada pelo Acute Physiology and Chronic Health Classification System II [Apache II]), tempo de ventilação mecânica invasiva (VMI) e tempo de sedação. A odds ratio (OR) foi estimada com intervalo de confiança de 95\%. Foram incluídos no estudo 92 pacientes sequenciais. Houve forte associação entre a não adoção de postura antigravitacional em UTI e óbito (ORajustada=37,7; IC=4,76-293; p=0,001). Conclui-se que pacientes que não adotaram postura antigravitacional durante o internamento em UTI apresentaram chances muito mais elevadas de mortalidade. Essa simples estratégia de classificação da capacidade funcional de pacientes críticos pode ser utilizada rotineiramente por equipes de saúde como uma variável simples e dicotômica de prognóstico de mortalidade em UTI.

Descritores | Cuidados Críticos; Unidades de Terapia Intensiva; Deambulação Precoce; Reabilitação; Terapia por Exercício.

ABSTRACT | Until now, few functional performance markers are able to predict death in Intensive Care Units (ICUs). This study aimed to identify the association between non-adoption of antigravity posture and death in patients admitted to an adult ICU. It is a retrospective and analytical study, performed through the analysis of medical records. Association between non-adoption of antigravity posture and death was tested by multiple logistic regression adjusted for gender, age, disease severity (measured by Acute Physiology and Chronic Health Classification System II [Apache II]), time of invasive mechanical ventilation (IMV), and period of sedation. The odds ratio (OR) with confidence interval ( $\mathrm{Cl}=95 \%)$ was estimated. A total of 92 sequential patients were included in the study. A strong association between the non-adoption of antigravity posture in the ICU and death (ORadjusted=37.7, Cl=4.76-293, p=0.001)

'Universidade Estadual do Sudoeste da Bahia (Uesb) - Jequié (BA), Brasil. E-mail: gil.fisio.2016@hotmail.com.

Orcid: 0000-0002-9692-3891

2Universidade Estadual do Sudoeste da Bahia (Uesb) - Jequié (BA), Brasil. E-mail: rofisio@hotmail.com. Orcid: 0000-0002-1366-3175

3Universidade Estadual do Sudoeste da Bahia (Uesb) - Jequié (BA), Brasil. E-mail: csjesus@uesb.edu.br. Orcid: 0000-0001-6727-3247

${ }^{4}$ Universidade Estadual do Sudoeste da Bahia (Uesb) - Jequié (BA), Brasil. E-mail: hitoef@uesb.edu.br. Orcid: 0000-0001-5095-0301

5Universidade Estadual do Sudoeste da Bahia (Uesb) - Jequié (BA), Brasil. E-mail: magno.uesb@gmail.com.

Orcid: 0000-0003-1375-2335

6Universidade Estadual do Sudoeste da Bahia (Uesb) - Jequié (BA), Brasil. E-mail: marcoshenriquefernandes@bol.com.br.

Orcid: 0000-0002-2559-2239

${ }^{7}$ Universidade Estadual do Sudoeste da Bahia (Uesb) - Jequié (BA), Brasil. E-mail: janilsonmatos@gmail.com.

Orcid: 0000-0003-4960-1010 
was observed. Thus, one can conclude that patients who did not adopt an antigravity posture during ICU admission had a much higher odds of mortality. This simple strategy to classify functional capacity of critical patients can be routinely used by the team as a simple and dichotomous variable for ICU mortality prognosis. Keywords | Critical Care; Intensive Care Units; Early Ambulation; Rehabilitation; Exercise Therapy.

RESUMEN | Aún existen pocos marcadores de desempeño funcional con capacidad de predecir la muerte en Unidades de Cuidados Intensivos ( $\mathrm{UCl}$ ). El presente estudio tuvo como objetivo identificar la asociación entre la no adopción de postura antigravitacional y el óbito en pacientes hospitalizados en una UCl adulto. Se trata de un estudio retrospectivo y analítico, realizado mediante análisis de historia clínica. La asociación entre la no adopción de postura antigravitacional y el fallecimiento fue probada por regresión logística múltiple ajustada por sexo, edad, gravedad de la enfermedad (medida por la Acute Physiology And Chronic Health Classification System II [Apache II]), tiempo de ventilación mecánica invasiva (VMI) y tiempo de sedación. Se estimó la odds ratio (OR) con intervalo de confianza (IC=95\%). Se incluyeron en el estudio 92 pacientes secuenciales. Se observó una fuerte asociación entre la no adopción de postura antigravitacional en UCI y el óbito (ORajustada=37,7; IC=4,76-293; $p=0,001)$. De esta forma, se puede concluir que pacientes que no adoptaron postura antigravitacional durante la hospitalización en UCI tuvieron posibilidades mucho más elevadas de mortalidad. Esta simple estrategia de clasificación de la capacidad funcional de pacientes críticos puede ser utilizada de manera rutinaria por el equipo de salud como una variable simple y dicotómica de pronóstico de mortalidad en UCl.

Palabras clave | Cuidados Críticos; Unidades de Cuidados Intensivos; Ambulación Precoz; Rehabilitación; Terapia por Ejercicio.

\section{INTRODUÇÃO}

A mobilização precoce, gradual e progressiva do paciente crítico internado em unidade de terapia intensiva (UTI) tem sido utilizada como uma estratégia de tratamento para evitar, minimizar ou reverter os efeitos deletérios do imobilismo prolongado no leito ${ }^{1}$. Para isso, foram desenvolvidos diversos protocolos com critérios rígidos de segurança, estratificados em níveis de mobilização que variam desde exercícios passivos até deambulação ${ }^{2-6}$.

Para que um paciente crítico realize movimentos executados contra a ação da gravidade e que envolvam carga axial da coluna ou ossos longos, como ocorre na sedestação, ortostase e deambulação, o fisioterapeuta deve-se assegurar de diversos critérios neurológicos, hemodinâmicos, ventilatórios e de oxigenação, relativos ao diagnóstico, a riscos de complicações e ao nível de suporte de vida, entre outros ${ }^{2-8}$.

A decisão de mobilizar pacientes contra a ação da gravidade envolve uma complexa análise multifatorial, que reflete a condição geral de saúde do paciente crítico $^{8-11}$. Assim, a adoção ou não de postura antigravitacional pode ser um bom marcador funcional, com capacidade preditiva de desfechos adversos em $\mathrm{UTI}^{12,13}$. Nesse sentido, o objetivo do presente estudo é verificar se existe associação entre não adoção de postura antigravitacional e óbito em pacientes internados em uma UTI adulto.

\section{METODOLOGIA}

Trata-se de um estudo retrospectivo e analítico, realizado na UTI adulto do Hospital Geral Prado Valadares (HGPV), em Jequié (BA), Brasil. A UTI desse hospital dispõe de 10 leitos, é classificada como geral (ou seja, atende a diversas especialidades clínicas), e possui serviço de fisioterapia com pelo menos um profissional fisioterapeuta durante 24 horas por dia, inclusive nos finais de semana.

Foram incluídos no estudo os pacientes admitidos na UTI durante o período de 15 de janeiro a 15 de maio de 2016, com tempo de internação maior do que 48 horas, e que tiveram registro de mobilização. Foram excluídos os pacientes que receberam alta, foram transferidos ou evoluíram a óbito com tempo menor ou igual a 48 horas e aqueles que não tiveram registro de mobilização em seu prontuário.

A variável dependente do estudo foi óbito durante a internação na UTI, e a variável independente foi a não adoção de postura antigravitacional durante a internação na UTI. A adoção ou não de postura antigravitacional foi categorizada a partir do protocolo adaptado de Morris et al. ${ }^{2}$, que é dividido em quatro níveis de mobilização:

- Nível 1: mobilizações passivas dos membros superiores (MMSS) e/ou inferiores (MMII);

- Nível 2: mobilizações ativo-assistidas de MMSS e/ou MMII, e/ou treino de transferência no leito e/ou exercícios de ponte de quadril; 
- Nível 3: Sedestação no leito, e/ou treino de equilíbrio de tronco e/ou transferência facilitada para a poltrona;

- Nível 4: Treino de equilíbrio em ortostase, e/ou atividades pré-marcha e/ou deambulação pela unidade.

Foram considerados casos de não adoção de postura antigravitacional os de pacientes que permaneceram apenas nos níveis 1 ou 2.

\section{Covariáveis}

Foram coletadas informações relacionadas a características demográficas e clínicas de cada paciente: idade (em anos completos), sexo (masculino ou feminino), diagnóstico (clínico ou cirúrgico), diagnóstico de sepse (não ou sim), gravidade da doença pontuada através do índice Acute Physiology and Chronic Health Evaluation II (Apache II), uso (não ou sim) e tempo de uso (em dias) de ventilação mecânica invasiva (VMI), uso (não ou sim) e tempo de uso (em dias) de sedação, tempo de internação (em dias) e desfecho (alta ou óbito).

O Apache II é utilizado para monitorar a gravidade da doença e o possível prognóstico do paciente crítico durante as primeiras 24 horas de admissão na UTI. A ferramenta é composta por 15 itens que pontuam sinais vitais, oxigenação, pH arterial, sódio, potássio, creatinina, hematócrito, leucócitos, bicarbonato (na ausência da gasometria), escala de coma de Glasgow e pontuação por idade e por doença crônica. $\mathrm{O}$ escore final varia de 0 a 71 pontos, e quanto mais alto o valor, maiores são as chances de mortalidade ${ }^{14}$.

\section{Análise estatística}

Foi realizada análise descritiva por meio de cálculo das frequências para variáveis categóricas, e média e desviopadrão para variáveis quantitativas. A associação entre não adoção de postura antigravitacional, covariáveis e óbito foi testada inicialmente por regressão logística binária (associação bruta), e permaneceram para análise ajustada apenas as variáveis que apresentaram $p \leq 0,20$. A análise ajustada foi realizada por meio da regressão logística múltipla (método de entrada stepwise forward), sendo estimada a odds ratio com intervalo de confiança (IC) de 95\%. As análises foram realizadas no software Package for the Social Sciences (SPSS) versão 21.0.

\section{RESULTADOS}

Durante o período de 15 de janeiro a 15 de maio de 2016 foram admitidos 127 pacientes na UTI do HGPV. Dessas pessoas, 92 obedeceram aos critérios de inclusão da pesquisa. A Figura 1 ilustra o fluxograma de distribuição dos pacientes.

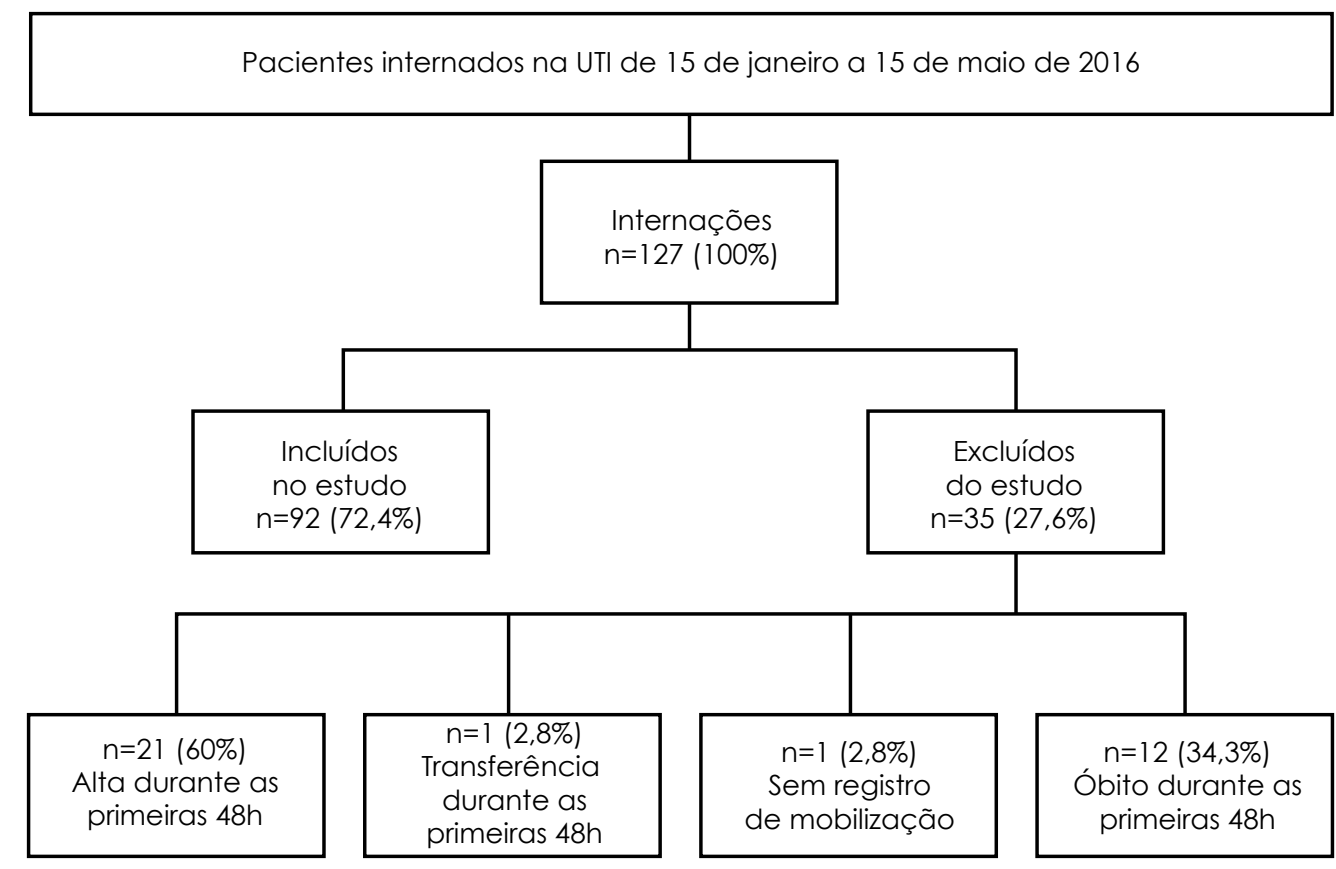

Figura 1. Fluxograma de distribuição dos pacientes. N: população total; n: amostra. 
A Tabela 1 apresenta as características demográficas e clínicas dos pacientes estudados e as variáveis categóricas e quantitativas. Pode-se observar que $51,1 \%$ da amostra foi constituída por homens, $67,4 \%$ dos diagnósticos primários foram de natureza clínica, $28,3 \%$ dos pacientes apresentaram sepse em algum momento da internação, e a gravidade da doença pontuada pelo Apache II admissional obteve uma média de $18,1( \pm 8,2)$ pontos. Dos pacientes, $79,3 \%$ utilizaram VMI e 76,1\% usaram sedação.

Tabela 1. Características demográficas e clínicas dos pacientes incluídos no estudo

\begin{tabular}{|c|c|c|c|c|}
\hline \multirow{3}{*}{ Variáveis categóricas } & \multirow{3}{*}{$\mathbf{N}(\%)$} & \multicolumn{3}{|c|}{ Óbito } \\
\hline & & \multicolumn{2}{|c|}{ Sim } & Não \\
\hline & & $\mathbf{n}$ & $\%$ & $\%$ \\
\hline \multicolumn{5}{|l|}{ Sexo } \\
\hline Feminino & $45(48,9)$ & 19 & 65,5 & 41,3 \\
\hline Masculino & $47(51,1)$ & 10 & 34,5 & 58,7 \\
\hline \multicolumn{5}{|l|}{ Diagnóstico (tipo) } \\
\hline Clínico & $62(67,4)$ & 22 & 75,9 & 36,5 \\
\hline Cirúrgico & $30(32,6)$ & 6) & 24,1 & 63,5 \\
\hline \multicolumn{5}{|l|}{ Uso de VMI } \\
\hline Sim & $73(79,3)$ & 28 & 96,6 & 71,4 \\
\hline Não & $19(20,7)$ & 7) & 3,4 & 28,6 \\
\hline \multicolumn{5}{|l|}{ Uso de sedação } \\
\hline Sim & $70(76,1)$ & 25 & 86,2 & 71,4 \\
\hline Não & $22(23,9)$ & 9) & 13,8 & 28,6 \\
\hline \multicolumn{5}{|l|}{ Postura antigravitacional } \\
\hline Não & $63(68,5)$ & 27 & 93,1 & 57,1 \\
\hline Sim & $29(31,5)$ & 5) & 6,9 & 42,9 \\
\hline \multirow{3}{*}{ Variáveis quantitativas } & & & \multicolumn{2}{|c|}{ Óbito } \\
\hline & \multirow{2}{*}{\multicolumn{2}{|c|}{$\begin{array}{l}\text { Média } \\
\text { (DP) }\end{array}$}} & Sim & Não \\
\hline & & & $\begin{array}{l}\text { Média } \\
\text { (DP) }\end{array}$ & $\begin{array}{l}\text { Média } \\
\text { (DP) }\end{array}$ \\
\hline Idade (anos completos) & & $54,1(19,0)$ & $60,4(14,2)$ & $51,2(20,2)$ \\
\hline Índice Apache II (pontos) & & $18,1(8,2)$ & $23,1(7,8)$ & $15,4(7,2)$ \\
\hline Tempo de VMI (dias) & & $10,5(10,8)$ & $14,4(11,1)$ & $8,8(10,3)$ \\
\hline Tempo de sedação (dias) & & $6,1(7,2)$ & $7,7(8,7)$ & $5,4(6,3)$ \\
\hline Tempo de internação na UT & (dias) & $11,8(8,9)$ & $12,7(8,2)$ & $11,4(9,3)$ \\
\hline
\end{tabular}

N: população total; n: amostra; VMI: ventilação mecânica invasiva; DP: desvio-padrão; Apache II: Acute Physiology and Chronic Health Evaluation II; UTI: unidade de terapia intensiva.

A Tabela 2 apresenta os resultados da associação bruta entre a não adoção de postura antigravitacional e óbito, assim como a associação bruta das covariáveis com óbito. Considerando um $p \leq 0,20$, permaneceram para análise ajustada as variáveis sexo, idade, Apache II, tempo de ventilação mecânica e tempo de sedação.

A análise ajustada (Tabela 3) continuou mostrando forte associação inversa entre a não adoção de postura antigravitacional e óbito, ou seja, indivíduos que não adotaram postura antigravitacional durante o internamento apresentaram maiores chances de evoluir a óbito ainda dentro da UTI.
Tabela 2. Associação bruta entre características clínicas e demográficas dos pacientes do estudo com óbito

\begin{tabular}{lrrr}
\multicolumn{1}{c}{ Variáveis } & \multicolumn{3}{c}{ Óbito } \\
\cline { 2 - 4 } & OR & IC 95\% & p valor \\
\hline $\begin{array}{l}\text { Não adotou postura } \\
\text { antigravitacional }\end{array}$ & 18,0 & $3,94-82,3$ & $<0,001$ \\
Sexo feminino & 2,70 & $1,08-6,75$ & 0,033 \\
Idade & 1,03 & $1,01-1,05$ & 0,034 \\
Diagnóstico clínico & 0,55 & $0,20-1,49$ & 0,243 \\
Índice Apache II & 1,15 & $1,07-1,23$ & $<0,001$ \\
Tempo de VMI (dias) & 1,05 & $1,01-1,09$ & 0,023 \\
Tempo de sedação (dias) & 1,04 & $0,98-1,11$ & 0,168 \\
Tempo de internação na UTI & 1,02 & $0,97-1,07$ & 0,519 \\
(dias) & & &
\end{tabular}

OR: odds ratio; IC: Intervalo de confiança; p valor: nível de significância; Apache II: Acute Physiology and Chronic Health Evaluation Il; VMI: ventilação mecânica invasiva; UTI: unidade de terapia intensiva.

Tabela 3. Resultado da regressão logística múltipla ajustada de associação entre não adoção de postura antigravitacional e óbito em pacientes internados em UTI

\begin{tabular}{lccr}
\multicolumn{1}{c}{ Variáveis } & \multicolumn{3}{c}{ Óbito } \\
\cline { 2 - 4 } \multicolumn{1}{c}{ Não adotou postura } & OR & IC 95\% & p valor \\
antigravitacional & 37,4 & $4,76-293$ & 0,001 \\
Sexo feminino & & & \\
Idade & 2,27 & $0,60-8,52$ & 0,226 \\
Índice Apache II & 1,01 & $0,97-1,05$ & 0,674 \\
Tempo de VMI (dias) & 1,16 & $1,05-1,28$ & 0,005 \\
Tempo de sedação (dias) & 1,08 & $0,99-1,17$ & 0,083 \\
& 0,98 & $0,87-1,11$ & 0,796
\end{tabular}

OR: odds ratio; IC: Intervalo de confiança; p valor: nivel de significância; Apache II: Acute Physiology and Chronic Health Evaluation Il; VMI: ventilação mecânica invasiva; UTI: unidade de terapia intensiva.

\section{DISCUSSÃO}

Este estudo demonstrou que, durante o internamento em UTI adulto, os pacientes que não adotaram postura antigravitacional apresentaram maiores chances de mortalidade. Logo, a postura antigravitacional pode ser uma estratégia simples de avaliação funcional, facilmente aplicável na prática clínica, e sua não adoção pode ser fator prognóstico de mortalidade em UTI. Esse é um resultado importante, que retrata a forte associação entre um indicador de performance funcional e mortalidade.

Já existem evidências de que a mobilização precoce, gradual e progressiva de pacientes internados em UTI reduz as chances de mortalidade. Isso se deve ao fato de que um programa de mobilização precoce, adequado e centrado em terapias ativas, melhora a capacidade funcional do paciente ${ }^{15}$ devido ao aumento da força dos músculos periféricos e respiratórios ${ }^{3}$, o que reduz o tempo de uso de $\mathrm{VMI}^{16,17}$, de internamento na unidade e no hospital ${ }^{17,18}$ e, consequentemente, as chances de complicações ${ }^{17,19}$ que podem agravar o quadro clínico do paciente ${ }^{7,13,20,21}$. 
A maioria dos estudos atuais sobre mobilização são ensaios clínicos controlados e randomizados, que comparam grupos de pacientes submetidos a protocolos específicos com cuidados-padrão de fisioterapia ${ }^{2,4,5,22}$.Tais estudos, portanto, apresentam um caráter intervencionista da mobilização. Sendo cada vez mais comum em UTIs, principalmente em níveis elevados e fora do leito ${ }^{14,23}$, a mobilização também pode ser vista como um fator prognóstico.

O presente estudo talvez seja o primeiro a apresentar a estratégia de mobilização dicotomizada (adoção ou não de postura gravitacional) com capacidade preditiva de mortalidade em UTI. Apesar de diversos estudos apresentarem a plausibilidade biológica ${ }^{10,24-26}$, ainda não havia sido demonstrada essa associação entre óbito e um indicador tão simples de capacidade funcional. Essa simples estratégia de classificação da capacidade funcional de pacientes críticos pode ser utilizada rotineiramente pela equipe como uma das variáveis de prognóstico de mortalidade em UTI.

Este estudo apresentou limitações referentes à indisponibilidade de dados sobre barreiras para mobilização que não fossem de origem estritamente relacionadas à gravidade da doença, como índice de massa corporal, por exemplo. Pacientes obesos podem não conseguir se sentar ou ficar de pé por questões mecânicas e necessidade de pessoal, e não necessariamente por um julgamento clínico baseado em critérios de gravidade.

Também não tivemos informações sobre o status funcional prévio dos pacientes. Pacientes já dependentes, com complicações funcionais prévias (por exemplo, contraturas e deformidades) podem apresentar maiores dificuldades para adotar postura antigravitacional.

Ainda assim, acreditamos que, mesmo com acesso a esses dados, haveria pouca interferência no processo analítico. Destaca-se que, embora este seja um estudo observacional e de análise de prontuário, a força da associação foi muito intensa, mesmo após o ajuste com variáveis que poderiam ser fatores de confusão, como Apache.

\section{CONCLUSÃO}

Este estudo demonstrou que pacientes que não adotaram postura antigravitacional durante o internamento em UTI apresentaram chances significativamente maiores de óbito. Essa simples estratégia de classificação da capacidade funcional pode ser utilizada rotineiramente pela equipe como uma das variáveis de prognóstico de mortalidade em UTI.

\section{REFERÊNCIAS}

1. Cameron S, Ball I, Cepinskas G, Choong K, Doherty TJ, Ellis $\mathrm{CG}$, et al. Early mobilization in the critical care unit: a review of adult and pediatric literature. J Crit Care. 2015;30(4):664-72. doi: 10.1016/j.jcrc.2015.03.032

2. Morris PE, Goad A, Thompson C, Taylor K, Harry B, Passmore L, et al. Early intensive care unit mobility therapy in the treatment of acute respiratory failure. Crit Care Med. 2008;36(8):2238-43. doi: 10.1097/CCM.0b013e318180b90e

3. Dantas CM, Silva PFS, Siqueira FHT, Pinto RMF, Matias S, Maciel C, et al. Influência da mobilização precoce na força muscular periférica e respiratória em pacientes críticos. Rev Bras Ter Intensiva. 2012;24(2):173-8. doi: 10.1590/ S0103-507X2012000200013

4. Feliciano V, Albuquerque CG, Andrade FMD, Dantas CM, Lopez A, Ramos FF, et al. A influência da mobilização precoce no tempo de internamento na Unidade de Terapia Intensiva. ASSOBRAFIR Ciênc. 2012;3(2):31-42

5. Carvalho TG, Silva ALG, Santos ML, Schäfer J, Cunha LS, Santos LJ. Relação entre saída precoce do leito na unidade de terapia intensiva e funcionalidade pós-alta: um estudo piloto. Rev Epidemiol Control Infect. 2013;3(3):82-6

6. Murakami FM, Yamaguti WP, Onoue MA, Mendes JM, Pedrosa RS, Maida ALV, et al. Evolução funcional de pacientes graves submetidos a um protocolo de reabilitação precoce. Rev Bras Ter Intensiva. 2015;27(2):161-9. doi: 10.5935/0103-507X.20150028

7. Hodgson C, Bellomo R, Berney S, Bailey M, Buhr H, Denehy $L$, et al. Early mobilization and recovery in mechanically ventilated patients in the ICU: a bi-national, multi-centre, prospective cohort study. Crit Care. 2015;19(1):81-91. doi: 10.1186/ s13054-015-0765-4

8. Harrold ME, Salisbury LG, Webb SA, Allison GT. Early mobilisation in intensive care units in Australia and Scotland: a prospective, observational cohort study examining mobilisation practises and barriers. Crit Care. 2015;19(1):336-9. doi: 10.1186/ s13054-015-1033-3

9. Kasotakis G, Schmidt U, Perry D, Grosse-Sundrup M, Benjamin J, Ryan C, et al. The surgical intensive care unit optimal mobility score predicts mortality and length of stay. Crit Care Med. 2012;40(4):1122-8. doi: 10.1097/CCM.0b013e3182376e6d

10. Sigler M, Nugent K, Alalawi R, Selvan K, Tseng J, Edriss H, et al. Making of a successful early mobilization program for a medical intensive care unit. South Med J. 2016;109(6):342-5. doi: 10.14423/SMJ.0000000000000472

11. Nydahl P, Ruhl AP, Bartoszek G, Dubb R, Filipovic S, Flohr HJ, et al. Early mobilization of mechanically ventilated patients: a 1-day point-prevalence study in Germany. Crit Care Med. 2014;42(5):1178-86. doi: 10.1097/CCM.0000000000000149

12. Herridge MS, Tansey CM, Matté A, Tomlinson G, Diaz-Granados N, Cooper A, et al. Functional disability 5 years after acute respiratory distress syndrome. N Engl J Med. 2011;364(14):1293304. doi: 10.1056/NEJMoa1011802 
13. Hermans G, Mechelen HV, Clerckx B, Vanhullebusch T, Mesotten D, Wilmer A, et al. Acute outcomes and 1-year mortality of ICUacquired weakness: a cohort study and propensity matched analysis. Am J Respir Crit Care Med. 2014;190(4):410-20. doi: 10.1164/rccm.201312-22570C

14. Knaus WA, Draper EA, Wagner DP, Zimmerman JE. APACHE II: a severity of disease classification system. Crit Care Med. 1985;13(10):818-29

15. Berney S, Haines K, Skinner EH, Denehy L. Safety and feasibility of an exercise prescription approach to rehabilitation across the continuum of care for survivors of critical illness. Phys Ther. 2012;92(12):1524-35. doi: 10.2522/ptj.20110406

16. Kayambu G, Boots R, Paratz J. Physical therapy for the critically ill in the ICU: a systematic review and metaanalysis. Crit Care Med. 2013;41(6):1543-54. doi: 10.1097/ cCM.0b013e31827ca637

17. McWilliams D, Weblin J, Atkins G, Bion J, Williams J, Elliott C, et al. Enhancing rehabilitation of mechanically ventilated patients in the intensive care unit: a quality improvement project. J Crit Care. 2015;30(1):13-8. doi: 10.1016/j.jcrc.2014.09.018

18. Hopkins RO, Mitchell L, Thomsen GE, Schafer M, Link M, Brown SM. Implementing a mobility program to minimize postintensive care syndrome. AACN Adv Crit Care. 2016;27(2):187203. doi: $10.4037 /$ aacnacc2016244

19. Hunter A, Johnson L, Coustasse A. Reduction of intensive care unit length of stay: the case of early mobilization.
Health Care Manag. 2014;33(2):128-35. doi: 10.1097/ HCM.0000000000000006

20. Stiller K. Physiotherapy in intensive care: an updated systematic review. Chest. 2013;144(3):825-47. doi: 10.1378/chest.12-2930

21. Laurent H, Aubreton S, Richard R, Gorce Y, Caron E, Vallat $A$, et al. Systematic review of early exercise in intensive care: a qualitative approach. Anaesth Crit Care Pain Med. 2016;35(2):133-49. doi: 10.1016/j.accpm.2015.06.014

22. Dong Z, Yu B, Sun Y, Fang W, Li L. Effects of early rehabilitation therapy on patients with mechanical ventilation. World J Emerg Med. 2014;5(1):48-52. doi: 10.5847/wjem.j.is sn.1920-8642.2014.01.008

23. Berney SC, Harrold M, Webb SA, Seppelt I, Patman S, Thomas PJ, et al. Intensive care unit mobility practices in Australia and New Zealand: a point prevalence study. Crit Care Resusc. 2013;15(4):260-5

24. Borges RC, Carvalho CR, Colombo AS, Borges MPS, Soriano FG. Physical activity, muscle strength, and exercise capacity 3 months after severe sepsis and septic shock. Intensive Care Med. 2015;41(8):1433-44. doi: 10.1007/s00134-015-3914-y

25. Iwashyna TJ, Cooke CR, Wunsch H, Kahn JM. Population burden of long-term survivorship after severe sepsis in older Americans. J Am Geriatr Soc. 2012;60(6):1070-7. doi: 10.1111/j.1532-5415.2012.03989.x

26. Williams PT. Inadequate exercise as a risk factor for sepsis mortality. PLoS One. 2013;8(12):1-7. doi: 10.1371/journal. pone.0079344 